\title{
Guglielmo Carchedi
}

\section{Die Ost-Erweiterung der EU}

Der EU-Erweiterung, die 2004 und 2007 stattgefunden hat, ${ }^{1}$ ging der Vertrag von Nizza voraus, der im Dezember 2000 unterzeichnet wurde. Die Botschaft, die von den Medien verbreitet wurde, lautete: Die EU muss Entscheidungsverfahren, die ursprünglich für eine Gemeinschaft von sechs Ländern aufgestellt wurden, an eine Gemeinschaft aus 25 oder mehr Länder anpassen, wenn sie nicht unregierbar werden soll. Tatsächlich waren die institutionellen Reformen aber notwendig geworden, um die Entscheidungsprozesse so umzuformen, dass es keine substanziellen Veränderungen der Machtverhältnisse zwischen den dominierenden und den dominierten Ländern geben würde. Aus dieser Perspektive soll der Vertrag von Nizza kurz beleuchtet werden. Der Vertrag ist momentan auch deshalb wichtig, weil er nach dem Scheitern der Referenden über eine EU-Verfassung in Frankreich und den Niederlanden das Funktionieren der EU reguliert.

Um die Interessen zu verstehen, die hinter den unterschiedlichen Verhandlungspositionen standen, die zu diesem Vertrag geführt haben, sind vier Punkte wichtig. Erstens: Die größeren Mitgliedsländer - Deutschland, England, Frankreich und Italien - versuchten ihre beherrschende Position zu verteidigen. Zweitens: Deutschland versuchte seine relative Vorherrschaft innerhalb dieses herrschenden Zirkels aufrecht zu erhalten oder auch zu verstärken. Drittens: Jedes der elf kleineren Länder fürchtete eine Schwächung seiner Position sowohl gegenüber den anderen Mitgliedsländern als auch gegenüber den Beitrittskandidaten. Viertens: Die Beitrittskandidaten wollten in der EU eine möglichst starke Position erreichen, insbesondere relativ $\mathrm{zu}$ den kleineren Mitgliedsländern.

Das Ergebnis, soweit es die Kommission betraf, bestand darin, dass Frankreich, England, Deutschland, Italien und Spanien jeweils einen ihrer beiden Kommissare verloren haben, so dass jedes Land nur noch einen Kommissar

1 Im Jahr 2004 traten der EU Estland, Lettland, Litauen, Tschechien, Polen, Ungarn, die Slowakei, Slowenien, Zypern und Malta bei. Sie werden im folgenden als EU-10 bezeichnet, im Unterschied zu den fünfzehn alten die Mitgliedsländern, die EU-15 genannt werden. Die ersten acht Länder der EU-10 werden als CEEC (Central and Eastern European Countries) bezeichnet. 2007 traten der EU außerdem noch Bulgarien und Rumänien bei. 
stellt. Dies ist in gewisser Weise ein Vorteil für die kleineren Länder. Außerdem wurde das Europäische Parlament von 626 auf 732 Abgeordnete vergrößert. Deutschland behielt seine 99 Abgeordneten, während die anderen Länder Sitze abgeben mussten. Damit hat Deutschland zwar sein relatives Gewicht im Parlament vergrößert, da das Parlament aber praktisch machtlos ist, bedeutet das nicht viel.

Worauf es ankommt, ist der Rat, das Gremium, das wirkliche Entscheidungsmacht hat. Deutschland verlangte als Konsequenz der Wiedervereinigung ein größeres Gewicht, vor allem Frankreich opponierte dagegen. In einigen besonders sensiblen Bereichen wie der Gemeinsamen Außen- und Sicherheitspolitik, der Steuerpolitik und der Asyl- und Immigrationspolitik müssen Entscheidungen nach wie vor einstimmig fallen. Aber auf den meisten Gebieten reicht jetzt eine qualifizierte Mehrheit. Dies erfordert eine Mehrheit der Mitgliedsstaaten und ein Minimum von 232 Stimmen. Allerdings kann eine Entscheidung blockiert werden, wenn diejenigen Staaten, die dagegen votierten, mindestens 38\% der EU Bevölkerung stellen. Es gibt 37 verschiedene Möglichkeiten wie drei Länder diese Sperrminorität erreichen können. Für 33 dieser Möglichkeiten ist Deutschland notwendig. Dies zeigt das gewachsene Gewicht von Deutschland, ohne Deutschland ist eine Sperrminorität nur sehr schwer zu erreichen. ${ }^{2}$ Man könnte nun meinen, Deutschland habe lediglich eine größere negative Macht gewonnen Entscheidungen zu blockieren, jedoch nicht die positive Macht Entscheidungen durchzusetzen. Aber diese negative Macht kann dazu benutzt werden, um im Austausch gegen die Aufgabe eines Veto Entscheidungen durchzusetzen: um so größer die Vetomacht, um so größer sind auch die daraus abgeleiteten Möglichkeiten der Durchsetzung. Insofern hat der Vertrag von Nizza die führende Position Deutschlands und damit auch des deutschen Kapitals gestärkt.

Vor diesem institutionellen Hintergrund muss man die ökonomischen Gründe für die Erweiterung sehen. In der kritischen Literatur wird üblicherweise argumentiert, dass die Erweiterung für die Versorgung mit Rohstoffen und Arbeitskräften gebraucht wurde und um weitere Investitionen aus der alten EU zu ermöglichen (Kagarlitsky 2004; Bohle 2006: 71-72). Es ist zwar richtig, dass die Osterweiterung es erleichtert diese Ziele zu erreichen, doch gibt es noch andere Gründe, aus denen die Erweiterung erfolgte. Um diese Gründe zu verstehen, ist es sinnvoll sich mit den Beziehungen der EU-15 und den CEEC vor der Erweiterung zu beschäftigen.

In der ersten Hälfte der 1990er Jahre zeigten die Beziehungen zwischen der EU und den CEEC im Vergleich mit dem klassischen Imperialismus neue Ei-

2 Dies erklärt auch warum man auf die Größe der Bevölkerung zurückgegriffen hat und nicht auf das Sozialprodukt. Dabei ergeben sich allerdings kuriose Effekte: Rumänien hat eine größere Stimmenzahl als Holland, dessen Sozialprodukt aber vier mal so groß ist. 
genschaften. Betrachten wir zunächst die Landwirtschaft. Im Zeitalter des klassischen Imperialismus (Kolonialismus) waren die abhängigen Länder Nettoexporteure von landwirtschaftlichen Produkten und Rohmaterialien und Nettoimporteure von industriellen Gütern, die aus den imperialistischen Zentren stammten. In den Zentren gab es eine Überproduktion von industriellen, aber nicht von landwirtschaftlichen Gütern. Neu ist nun, dass in den dominanten Ländern der EU die Überproduktion auch die Landwirtschaft erfasst hat: inzwischen wurde die EU auch zum Nettoexporteur von agrarischen Gütern. So entwickelte sich die EU zwischen 1991 und 1996 aus einem Nettoimporteur von Nahrungsmitteln, Getränken und Tabak aus den CEEC im Umfang von 0,5 Mrd. ECU zu einem Nettoexporteur im Umfang von 2 Mrd. ECU. Andererseits blieb die klassische koloniale Struktur bei Brennstoffen, Schmiermitteln und ähnlichen Materialien bestehen (Carchedi 2001: 183).

Betrachtet man Industriegüter, dann scheint es zunächst so als würde die koloniale Struktur fortbestehen. Zwischen 1991 und 1996 hat sich der Export solcher Güter aus der EU in die CEEC nahezu vervierfacht und der Überschuss der EU in diesem Bereich machte einen gewaltigen Sprung von 3 Mrd. ECU auf 19,2 Mrd. ECU. Betrachtet man einzelne Länder, sieht man, dass sich bei Polen ein Überschuss von 604 Mill. ECU (1988) in ein Defizit von 7,6 Mrd. ECU (1996) verwandelt hat, in der selben Periode wurde in Rumänien aus einem Überschuss von 1,6 Mrd. ECU ein Defizit von 800 Mill. ECU und Ungarn steigerte sein Defizit von 196 Mill. ECU auf 1,2 Mrd. ECU (Carchedi 2001: 184). Untersucht man jedoch einzelne Sektoren, dann wird deutlich, dass es sich eher um die Struktur abhängiger Entwicklung handelt, die hier eine Rolle spielt. Das Handelsbilanzdefizit der EU bei verschiedenen Industriegütern wird mehr als ausgeglichen durch den Überschuss bei chemischen Produkten und Maschinen, d.h. durch Hochtechnologiegüter. Während die CEEC ihre Exporte solcher Güter zwar auch steigern konnten, stiegen ihre Importe dieser Güter aber weit stärker an. Ein Handelsbilanzüberschuss von 5,4 Mrd. ECU (1991) in diesem Bereich verwandelte sich in ein Defizit von 19,5 Mrd. ECU (1996). Aber selbst mit diesen Zahlen wird die Exportkapazität der CEEC noch überschätzt, denn ein Teil ihrer Exporte besteht lediglich aus Zwischenprodukten die an Unternehmen aus der EU geliefert werden. Bei den Dienstleistungen ist die Situation weniger eindeutig. Die CEEC wurden in diesem Bereich zu einem wichtigen Handelspartner der EU, wobei die Dienstleistungsbilanz der EU zunächst einen kleinen Überschuss, später ein kleines Defizit aufwies.

Bei den Ausländischen Direktinvestitionen (ADI) ist die imperialistische Struktur sehr deutlich. 1995 betrugen die Direktinvestitionen der EU in den CEEC 5,6 Mrd. ECU, das waren 12,6\% der gesamten Kapitalexporte, die die EU verließen. ADI in umgekehrter Richtung spielten keine Rolle. Die mit Abstand am meisten Investitionen kamen aus Deutschland (38\%), gefolgt von 
Frankreich, den Niederlanden und Österreich. Aus diesen Ländern kamen $82 \%$ der ADI, die aus der EU in die CEEC flossen.

Die Struktur der Abhängigkeit der 2004 beigetretenen zehn neuen Mitglieder (EU-10) setzt sich auch im 21. Jahrhundert fort. Wie Tabelle 1 zeigt sind BIP, Importe und Exporte beträchtlich gewachsen

Tabelle 1: EU-10: Wachstum des BIP, der Importe und Exporte in Prozent

\begin{tabular}{|l|l|l|l|l|}
\hline & 2002 & 2003 & 2004 & 2005 \\
\hline (1) BIP & 2.4 & 4.0 & 4.9 & 4.5 \\
\hline (2) Importe & k.A. & 26.1 & 28.5 & k.A. \\
\hline (3) Exporte & k.A. & 29.1 & 30.6 & k.A. \\
\hline
\end{tabular}

Quelle: UNECE (2005, table 1.1.1, S.2; table 6.2.1 S.93)

Es muss betont werden, dass diese hohen Wachstumsraten in Zusammenhang mit den erheblichen Verwüstungen der Ökonomien der CEEC in der Transformationsperiode stehen und sich nicht etwa den schöpferischen Leistungen eines entfesselten Kapitalismus verdanken. Diese hohen Wachstumsraten passen zur abhängigen Rolle dieser Länder innerhalb ihrer imperialistisch strukturierten Beziehung zur EU. Die Tabellen 2 und 3 machen diesen Punkt deutlich.

Tabelle 2: EU-10: Bilanzsalden in Prozent vom BIP

\begin{tabular}{|l|l|l|l|}
\hline & 2002 & 2003 & 2004 \\
\hline (1) Handelsbilanzsaldo & k.A.. & -7.0 & -7.1 \\
\hline (2) Dienstleistungsbilanzsaldo & -3.1 & -3.0 & -3.0 \\
\hline (3) Leistungsbilanzsaldo & -4.3 & -4.4 & -5.0 \\
\hline (4) Zufluss an ADI & 5.5 & 2.5 & 3.1 \\
\hline
\end{tabular}

Quelle: UNECE (2005: table 6.2.1, S. 93; table 6.1.2, S.86)

Betrachten wir nun die EU-25 wie in Tabelle 3. Seit dem Jahr 2000 haben die ADI einen dramatischen Rückgang erlebt. Die von den EU-25 getätigten ADI außerhalb der EU-25 sind von 436 Mrd. Euro im Jahre 2000 (nicht in der Tabelle enthalten) auf $126 \mathrm{Mrd}$. Euro im Jahr 2004 zurückgegangen (Zeile 1). Die in den EU-25 getätigten ADI sind ebenfalls zurückgegangen, von 180 Mrd. Euro im Jahr 2000 (nicht in der Tabelle), auf 54 Mrd. im Jahr 2004 (Zeile 4). Dabei spielten aber sowohl für die von außerhalb der EU-25 eingehenden ADI, wie für die ADI, welche die EU-25 verlassen, die „alten“ Mitgliedsländer (die EU-15) die entscheidende Rolle, wie aus den Zeilen 2 und 5 deutlich wird. 
Tabelle 3: In die EU-25 eingehende bzw. sie verlassende ADI in Mrd. Euro

\begin{tabular}{|l|l|l|l|l|}
\hline & 2001 & 2002 & 2003 & 2004 \\
\hline $\begin{array}{l}\text { (1) Von der EU-25 getätigte, die } \\
\text { EU-25 verlassende ADI }\end{array}$ & 286.4 & 127.7 & 132.5 & 126.3 \\
\hline (2) - aus der EU-15 & 285.5 & 127.5 & 131.0 & 125.0 \\
\hline (3) - aus der EU-10 & 0.9 & 0.2 & 1.5 & 1.3 \\
\hline $\begin{array}{l}\text { (4) In der EU-25 getätigte ADI } \\
\text { von außerhalb der EU-25 }\end{array}$ & 120.1 & 139.8 & 125.2 & 54.1 \\
\hline (5) - in die EU-15 & 113.3 & 136.6 & 122.5 & 50.7 \\
\hline $\begin{array}{l}\text { (6) - in die EU-10 } \\
\text { (7) Nettozuflüsse von ADI in die }\end{array}$ & 2.9 & 3.2 & 2.6 & 3.4 \\
\hline
\end{tabular}

Quelle : Passerini (2005)

Die ADI, die zwischen den EU-Mitgliedern fließen, zeigt Tabelle 4

Tabelle 4: ADI innerhalb der EU in Mrd. Euro

\begin{tabular}{|l|l|l|l|l|}
\hline & 2001 & 2002 & 2003 & 2004 \\
\hline (1) ADI innerhalb der EU-25 & 381.6 & 348.4 & 250.3 & 156.5 \\
\hline (2) Von den EU-15 in die EU-10 & 18.8 & 13.0 & 7.0 & 10.6 \\
\hline (3) Von den EU-10 in die EU-15 & 1.0 & 1.1 & 0.8 & 3.4 \\
\hline (4) Zwischen den EU-15 & 361.4 & 335. & 241.9 & 141.7 \\
\hline (5) Zwischen den EU-10 & 0.4 & 0.9 & 0.7 & 0.8 \\
\hline (6) Nettozuflüsse in die EU-10 & 17.8 & 11.9 & 6.2 & 7.2 \\
\hline
\end{tabular}

Quelle: Passerini (2005)

Die EU-15 vereinigt auf sich nicht nur fast die gesamten außerhalb der EU-25 getätigten und in die EU-25 einfließenden ADI (vgl. Tabelle 3), sie vereinigt auf sich auch fast die ganzen innerhalb der EU-25 getätigten bzw. einfließenden ADI (vgl. Tabelle 4 Zeile 4), die CEEC spielen nur eine untergeordnete Rolle als Empfänger von ADI (Tabelle 4 Zeile 3). Die Abhängigkeitsstrukturen werden deutlich, wenn wir Zeile 7 in Tabelle 3 mit Zeile 6 in Tabelle 4 vergleichen: die ADI, die die EU-10 aus den EU-15 erhalten sind bei weitem größer als die ADI von außerhalb der EU-15. Dies ist ein deutliches Anzeichen dafür, dass die imperialistischen Abhängigkeitsverhältnisse der EU-10 zu Ländern innerhalb der EU weit größer sind als zu Ländern außerhalb.

Sofern wir den Handel und die Investitionen betrachten, zeigt sich, dass es zwischen der EU-15 (insbesondere den dort dominierenden Ländern) und den EU-10 eine imperialistische Abhängigkeit („Handels- und Finanzintegration“ in der Sprache der Mainstream-Ökonomie) gibt. Diese Beziehung existierte schon vor der Erweiterung und sie hätte auch ohne EU-Erweiterung fortbestanden. Von der Erweiterung war in dieser Hinsicht kein bedeutender Vorteil zu erwarten. Dies gilt insbesondere für die dominierenden Länder der EU-15 
und hier besonders für Deutschland: bereits 1996 exportierte Deutschland mehr in die CEEC als in die USA (Gowan 1997). Dasselbe trifft für eine den Interessen der dominanten EU-Kapitale entsprechende Restrukturierung der ökonomischen und sozialen Institutionen der CEEC zu. Eine solche Restrukturierung wurde bereits durch den IWF und die Weltbank ermöglicht, in deren Klauen die CEEC schon in den 1990er Jahren gefallen waren. Und schließlich erhalten wir das selbe Ergebnis auch für den Zugriff auf die Arbeitskraft. Mit der Erweiterung mögen sich zwar die formalen Bedingungen der Regulation ändern, aber der Inhalt des Prozesses bleibt der selbe: die Versorgung mit billiger Arbeitskraft entsprechend den Bedürfnissen der dominanten Kapitale und ein konsequenter Druck auf die Lebens- und Arbeitsbedingungen (einschließlich der Reallöhne) der Arbeiter und Arbeiterinnen in der EU. ${ }^{3}$

Sicher kann die Erweiterung die bestehenden Vorteile für die führenden Kapitale in den dominierenden Ländern der EU vergrößern. Aber dies reicht als Erklärung für die Erweiterung kaum aus. Es gibt andere Gründe für die Erweiterung, die mit der spezifischen Phase des gegenwärtigen Imperialismus zu tun haben, d.h. mit dem Entstehen einer Reihe von Staaten, denen gegenüber die USA zwar nach wie die überlegene Militärmacht darstellen, doch sind die USA längst nicht mehr die absolut dominierende Wirtschaftsmacht. Vor diesem Hintergrund spielen vor allem drei Bereiche für die EU-Erweiterung eine wichtige Rolle.

Erstens gibt es geopolitische Gründe. Von herausragender Bedeutung ist die Konkurrenz zwischen den USA und der EU um die Ölversorgung und die Diversifizierung der Quellen, aus denen die Ölversorgung stammt. Eine wichtige Pipeline verläuft vom Kaspischen Meer durch Ungarn hindurch um Westeuropa zu versorgen. Außerdem haben einige Länder spezifische Interessen. Deutschland z.B. war unter anderem deshalb an der Osterweiterung interessiert, weil es mit Polen und Tschechien direkte Grenzen hat und diese Länder einen Puffer nach Osten bilden sowie eine periphere Region, aus der leicht Ressourcen geschöpft werden können.

Zweitens sollte die Erweiterung im Kontext der militärischen Konkurrenz zwischen den USA und der EU gesehen werden (Carchedi 2001). Die USA haben ihren Einfluss auf die CEEC durch deren NATO-Beitritt ausgedehnt. Aber dieselben Länder müssen sich als EU-Mitglieder auch an der Sicherheits- und Verteidigungspolitik der EU beteiligen, die im Gegensatz zu den offiziellen Verlautbarungen durchaus eine Alternative zur NATO darstellt (vgl. Carchedi 2006). Trotzdem haben die USA eine positive Haltung zur EU-Erweiterung, denn die Sicherheits- und Verteidigungspolitik der EU ist lediglich eine sehr

3 Aufgrund innenpolitischer Erwägungen haben viele der EU-15 Länder die Zuwanderung von Arbeitskräften aus den EU-10 für eine "Übergangsperiode" beschränkt. Berücksichtigt man, dass viele der aus den CEEC stammenden Zuwanderer höher qualifizierte Arbeitskräfte sein dürften, ist dort mit einem erheblichen brain drain zu rechnen. 
langfristige Drohung für die militärische Macht der USA. Mit ihrer NATO Mitgliedschaft und der proamerikanischen Haltung ihrer neuen Eliten sind die CEEC im Moment eher eine Schwächung für die politische Einheit einer erweiterten EU.

Drittens ist die Erweiterung der erste Schritt, um den Euro in den neuen Mitgliedsländern einzuführen. Dies ist ein wichtiger Gesichtspunkt in der Konkurrenz zwischen Euro und Dollar um die internationale Seignorage, d.h. die Vorteile aus der Position als Weltgeld. Damit aber eine Währung die Rolle des Weltgeldes übernehmen kann, müssen zwei Bedingungen erfüllt sein: sie muss in einem weiten Bereich als internationales Zahlungsmittel benutzt werden und sie muss als Reservewährung dienen, wofür ihr Wert weitgehend konstant bleiben oder steigen sollte (vgl. Carchedi 2005).

Was die erste Bedingung betrifft, so haben sich die neuen Mitgliedsländer dazu verpflichtet den Euro möglichst bald einzuführen, Slowenien hat dies bereits am 1. Januar diesen Jahres getan. Wenn der Euro in den neuen Mitgliedsländern eingeführt ist, werden alle Transaktionen in der vergrößerten Eurozone in Euro abgewickelt werden und auch die Exporte der neuen Mitgliedsländer in Länder außerhalb der EU müssen dann in Euro bezahlt werden. Ein konstanter oder steigender Wert des Euro, der nötig ist, damit der Euro seine Rolle als internationale Reservewährung ausbauen kann, wird letzten Endes von einer konstanten oder steigenden Handelsbilanz abhängen. Im Jahr 2002 hatten die EU-15 mit dem Rest der Welt einen Handelsbilanzüberschuss von 6,3 Mrd. Euro. Die 10 im Jahre 2004 beigetretenen Länder hatten damals aber ein erhebliches Handelsbilanzdefizit gegenüber dem Rest der Welt (Cristallo 2003). Hätten die EU-10 schon 2002 zur EU gehört und den Euro benutzt, dann hätte die (damals fiktive) EU-25 gegenüber dem Rest der Welt ein enormes Handelsbilanzdefizit aufgewiesen, was den Euro als internationale Währung erheblich geschwächt hätte.

Damit die Einführung des Euro in den neuen Mitgliedsländern dem Euro auf seinem Weg zum Weltgeld hilft, muss deren Handelsbilanz mit den Nicht-EU Ländern positiv werden. Zu diesem Zweck muss ihre ökonomische Struktur weiter verändert werden, so dass sie zu Nettoexporteuren werden können. Dies erfordert eine Vertiefung der neoliberalen Politik. ${ }^{4}$ Dies ist der Grund warum die dominanten Länder der EU zwar die Einführung des Euro in den neuen Mitgliedsländern befürworten, aber nur dann, wenn diese darauf „vorbereitet“ sind. Allerdings dürften dort nach der Einführung des Euro Handelsbilanzüberschüsse eher schwieriger zu erreichen sein, denn abgesehen von ihrem

4 Genauer gesagt ist erstens eine Politik erforderlich, die den inneren Markt vergrößert und auf ADI aus den führenden Ländern der EU setzt, was den Export begünstigt. Zweitens wird die noch vom Staat regulierte Sphäre weiter privatisiert und dereguliert werden müssen. Wenn es unter diesen Umständen zu einer Entwicklung der Beitrittsländer kommt, wird es eine abhängige Entwicklung sein. 
technologischen Rückstand zu den führenden Ländern der EU haben sie dann auch nicht mehr die Möglichkeit ihre Konkurrenzfähigkeit durch Währungsabwertung oder eine Veränderung der Leitzinsen (die dann von der EZB festgelegt werden) zu erreichen.

Die Konsequenzen für die Arbeiterklasse in der EU können nur negativ sein, und zwar aus zwei Gründen. Erstens, die Ideologen der herrschenden Klasse behaupten, dass die Einkommensdifferenzen zwischen den alten und den neuen Mitgliedsländern abnehmen werden, so wie es bei Spanien, Portugal und Griechenland nach ihrem EU-Beitritt auch der Fall war. Aber dieser Vergleich ist falsch. Wie Kagarlitsky (2004) betonte, spielten sich die früheren Erweiterungen in einem anderen Kontext ab. Es herrschte noch der Kalte Krieg, so dass aus politischen Gründen eine Politik der Umverteilung notwendig war. Auch war es angesichts der militärischen Übermacht der USA unvorstellbar für die europäischen Länder eine ähnliche Militärmacht anzustreben. Der Zusammenbruch der Sowjetunion machte dann aber eine Politik, die die Bedürfnisse der Arbeiterklasse berücksichtigte, weniger notwendig. Aus diesen Gründen werden die neuen EU-Mitglieder nicht mehr die selben Vorteile genießen wie sie früher die ärmeren Staaten der EU erfahren haben und genauso wenig wird die Arbeiterklasse dieser Staaten noch von einer Umverteilungspolitik der EU oder ihrer eigenen Staaten profitieren können. ${ }^{5}$

Zweitens, wie schon oben erwähnt, ist es notwendig für die Stärkung der Rolle des Euro als internationale Währung, dass die neuen Mitglieder Nettoexporteure werden. Dies ruft nach neoliberalen Strategien wie Einschnitten in den Sozialstaat, der Privatisierung staatlicher Unternehmen und der Abwicklung unprofitabler industrieller und landwirtschaftlicher Bereiche. Aufgrund von diesem und dem zuvor angeführten Punkt wird es die europäische Arbeiterklasse sein, die für den „Erfolg“ der Erweiterung bezahlen muss.

Übersetzung aus dem Englischen von Michael Heinrich

\section{Literatur}

Bohle, D. (2006): Neo-liberal hegemony, transnational capital and the terms of the EU's eastward expansion, Capital and Class, Nr.88, Spring 2006, S. 57-86

Carchedi, G. (2006): Der militärische Arm der Europäischen Union, in: PROKLA 144, S. 381-396

Carchedi, G. (2001): For another Europe, Verso, London

Carchedi, G. (1991): Frontiers of Political Economy, Verso, London

Carchedi, G. (2005): Il contraddittorio cammino dell'Europa verso l'Est, in L.Vasapollo (Hg.), S. 217-239

Cristallo, D. (2003): Trade in a 25-Member European Union, Statistics in Focus, Eurostat, Luxemburg

Gowan, P. (1997): The Dynamics of European Enlargement, Labour Focus on Eastern Europe, No. 56

5 Die neuen Mitglieder werden von der EU zwar erhebliche Beträge erhalten, diese dienen aber vor allem der Verbesserung der Infrastruktur. 
Hoof, K.; Stiglitz, J.E. (2002): After the Big Bang?, Policy Research Working Paper 2934, The World Bank

Kagarlitsky, B. (2004): Is European Division Really Over?, http://www.tni.org/archives/ kagarlitsky/division.htm

Passerini, P. (2005): Decreased FDI flows for the EU25 in 2004, Eurostat, Statistics in Focus, $32 / 2005$

UNECE (2005): Economic Survey of Europe, No. 1

Vasapollo, L. (2005) (ed.): Lavoro contro Capitale, Jaca Books, Mailand

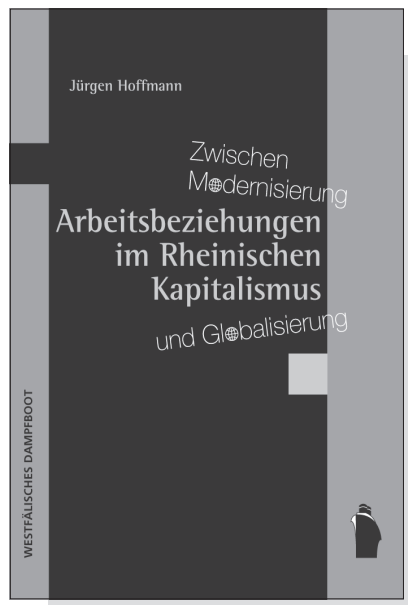

Jürgen Hoffmann

Zwischen Modernisierung und Globalisierung

Arbeitsbeziehungen im

Rheinischen Kapitalismus

2006 - 211 Seiten - € 19,90

ISBN 978-3-89691-644-0 